(n)

\title{
Automated recognition and
} quantification of pancreatic islets in Zucker diabetic fatty rats treated with exendin-4

\author{
Tetsuhiro Kakimoto', Hirotaka Kimata ${ }^{2}$, Satoshi Iwasaki ${ }^{2}$, Atsushi Fukunari ${ }^{1}$ and \\ Hiroyuki Utsumi ${ }^{1}$
}

${ }^{1}$ Safety Research Laboratory and ${ }^{2}$ Department I, Pharmacology Research Laboratories II, Research Division, Mitsubishi Tanabe Pharma Corporation, 2-2-50, Kawagishi, Toda-shi, Saitama 335-8505, Japan
Correspondence should be addressed to T Kakimoto Email

kakimoto.tetsuhiro@ mf.mt-pharma.co.jp

\begin{abstract}
Type 2 diabetes is characterized by impaired insulin secretion from pancreatic $\beta$-cells. Quantification of the islet area in addition to the insulin-positive area is important for detailed understanding of pancreatic islet histopathology. Here we show computerized automatic recognition of the islets of Langerhans as a novel high-throughput method to quantify islet histopathology. We utilized state-of-the-art tissue pattern recognition software to enable automatic recognition of islets, eliminating the need to laboriously trace islet borders by hand. After training by a histologist, the software successfully recognized even irregularly shaped islets with depleted insulin immunostaining, which were quite difficult to automatically recognize. The results from automated image analysis were highly correlated with those from manual image analysis. To establish whether this automated, rapid, and objective determination of islet area will facilitate studies of islet histopathology, we showed the beneficial effect of chronic exendin-4, a glucagon-like peptide-1 analog, treatment on islet histopathology in Zucker diabetic fatty (ZDF) rats. Automated image analysis provided qualitative and quantitative evidence that exendin- 4 treatment ameliorated the loss of pancreatic insulin content and gave rise to islet hypertrophy. We also showed that glucagon-positive $\alpha$-cell area was decreased significantly in ZDF rat islets with disorganized structure. This study is the first to demonstrate the utility of automatic quantification of digital images to study pancreatic islet histopathology. The proposed method will facilitate evaluations in preclinical drug efficacy studies as well as elucidation of the pathophysiology of diabetes.
\end{abstract}

$\begin{aligned} & \text { Key Words } \\ & \text { - } \text { automated image analysis } \\ & \text { - Zucker diabetic fatty rat } \\ & \text { - islet of Langerhans } \\ & \text { - insulin-positive } \beta \text { cell } \\ & \text { - exendin-4 }\end{aligned}$

Journal of Endocrinology (2013) 216, 13-20

\section{Introduction}

Type 2 diabetic patients are dramatically increasing in number and diabetes has become a global health problem. Impaired insulin secretion and insulin resistance are characteristic features of diabetes mellitus (Prentki \& Nolan 2006). $\beta$-Cell mass in the islets of Langerhans is decreased in diabetic patients, while obese subjects without diabetes have increased $\beta$-cell mass and insulin secretion to compensate for insulin resistance (Sakuraba et al. 2002, Butler et al. 2003, Yoon et al. 2003), suggesting that subjects with insulin resistance develop diabetes with the onset of $\beta$-cell dysfunction. 
Drugs to effectively increase insulin-secreting $\beta$-cells have been long-awaited. Glucagon-like peptide-1 (GLP-1), a member of the incretin family, has attracted much interest because of its multiple anti-diabetic actions (Drucker 2006, Drucker \& Nauck 2006). GLP-1 is secreted from intestinal L-cells in response to nutrient intake and acts to stimulate insulin secretion from $\beta$-cells. GLP- 1 and its long-acting analog exendin-4 (Kolterman et al. 2003) have been suggested to expand the insulin store in $\beta$-cells. Several reports have demonstrated an increase in pancreatic insulin-positive $\beta$-cells by exendin- 4 treatment in animal models of type 2 diabetes (Xu et al. 1999, Stoffers et al. 2000, Tourrel et al. 2001, Wang \& Brubaker 2002, Li et al. 2003). Here, we have investigated beneficial effects of chronic exendin-4 treatment on glycemic control and pancreatic islet histology in Zucker diabetic fatty (ZDF) rats, which has not been sufficiently investigated (Finegood et al. 2001).

The ZDF rat strain is of increasing preclinical interest, because of its pathophysiological similarities to human type 2 diabetes (Clark et al. 1983, Tokuyama et al. 1995, Pick et al. 1998, Jones et al. 2010). ZDF rats are characterized by a leptin receptor mutation leading to hyperphagia, hyperleptinemia, insulin resistance, and obesity. ZDF rats are euglycemic at 5-6 weeks of age but develop diabetes at about 10 weeks, becoming increasingly hyperglycemic and hypoinsulinemic with progressive islet failure. Pancreatic islets of ZDF rats show adaptive hypertrophy to overcome insulin resistance. However, diabetic ZDF islets have insulin immunostaining with variable intensity, suggesting evidence of insulin degranulation. Therefore, quantification of the islet area in addition to the insulinpositive area is important for detailed understanding of pancreatic islet histopathology. Although the quantification of the insulin-positive $\beta$-cell area is quite common, there have been few reports that quantified islet area in addition to insulin-positive $\beta$-cell area.

One of the biggest challenges in obtaining accurate results in the image analysis of islet area is the segregation of islets from other tissues on a slide. In the case of normal islets with homogeneous insulin immunostaining, the value of insulin-stained area that can be quantified easily using automatic isolation of colored pixels (Rojo et al. 2009, Prasad \& Prabhu 2011, Isse et al. 2012) is very similar to that of the islet area. However, the area of ZDF islets with depleted insulin immunostaining cannot be quantified by the colored pixel-based measurement. Manual tracing of target regions will provide accurate results, but this method is quite labor intensive and time consuming, especially for regions as irregularly shaped as ZDF islets.
In this study, state-of-the-art tissue pattern recognition software enabled automatic recognition and quantification of pancreatic islets, eliminating the need to laboriously trace islet borders by hand. To establish whether this automated, rapid, and accurate determination of pancreatic insulin-positive area and islet area will facilitate studies of islet histopathology, we investigated the potential of exendin- 4 treatment to beneficially affect islet pathology in diabetic ZDF rats. This study is the first, to our knowledge, to apply automated recognition-based quantitative image analysis to study pancreatic islet histopathology.

\section{Materials and methods}

\section{Animals}

Male rats were purchased from Charles River Laboratories Japan, Inc. (Yokohama, Japan). The animals were maintained on a standard laboratory chow diet under a $12 \mathrm{~h}$ light:12 h darkness cycle. Sixteen ZDF rats (Lepr ${ }^{f a} /$ Lepr $^{f a}$ ) were randomly divided into a vehicle-treated control group or an exendin-4-treated group (eight animals each). At 5 weeks of age, ZDF rats were subjected to a continuous s.c. infusion of PBS as vehicle or exendin-4 (synthesized by Operon Biotechnology, Tokyo, Japan) for 8 weeks using Alzet osmotic pumps (Durect Corp., Cupertino, CA, USA), which were set to deliver over $10 \mu \mathrm{g} / \mathrm{kg}$ body weight per day of exendin-4. Additionally, eight ZDF lean (ZL) rats $\left(+/\right.$ Lepr $^{f a}$ or $\left.+/+\right)$ were examined as a non-diabetic control. All animal experiments were in accordance with the institutional guidelines and were approved in advance by the Committee of Animal Experiments in the Research Division of Mitsubishi Tanabe Pharma Corporation.

\section{Blood biochemistry}

Blood samples were collected from the tail vein and HbA1c levels were determined by a HbA1c analyzer (Tosoh, Tokyo, Japan), according to the manufacturer's instructions. Plasma fraction was separated by centrifugation. Plasma glucose levels were determined by glucose CII-test Wako (Wako, Osaka, Japan) and a microplate reader (Molecular Devices, Sunnyvale, CA, USA), according to the manufacturer's instructions. Plasma insulin levels were determined by an Ultra Sensitive Rat Insulin ELISA Kit (Morinaga Institute of Biological Science, Yokohama, Japan), according to the manufacturer's instructions.

Published by Bioscientifica Ltd. 


\section{Histology and immunohistochemistry}

At 13 weeks of age, rats were killed with $20 \mathrm{mg}$ of i.p. Nembutal. The pancreas was removed from each animal and immediately fixed in $10 \%$ neutralized buffered formalin for histological analysis. Formalin-fixed pancreases were minced to $5 \mathrm{~mm}$ to randomize the observation area, and embedded in paraffin. Paraffin sections were stained with hematoxylin and eosin.

Immunohistochemistry was performed as described previously (Yamairi et al. 2011). Briefly, pancreas paraffin sections were deparaffinized. For insulin immunostaining, sections were incubated overnight at $4{ }^{\circ} \mathrm{C}$ with anti-insulin guinea pig polyclonal antibody (Dako, Glostrup, Denmark) followed by HRP-conjugated secondary antibody (antiguinea pig IgG goat polyclonal antibody; Millipore, Billerica, MA, USA). For glucagon and somatostatin, sections were incubated overnight at $4{ }^{\circ} \mathrm{C}$ with anti-glucagon and antisomatostatin rabbit polyclonal antibody (Dako), respectively, followed by HRP-conjugated secondary antibody (anti-rabbit IgG goat polyclonal antibody; Nichirei, Tokyo, Japan). The sections were stained with 3,3'-diaminobenzidine. The sections were photographed with an Olympus DP73 digital camera system and cellSens Software equipped with an Olympus BX51 microscope.

\section{Image analysis}

Whole slide digital images of the sections immunostained for insulin were obtained with Scan Scope XT (Aperio, Vista, CA, USA). Islet area, insulin-positive area, and pancreas area were automatically quantified using Tissue Studio (Definiens, Munich, Germany). Briefly, whole slide images were loaded with resolution and the specimen was automatically separated from the background glass area. The included 'composer training tool' was used to train the software to identify the islet area after segmentation with the scale set to 6 . The immunopositive area was quantified using the supplied 'marker area detection tool' with a threshold of 0.5 . Once the algorithm was tuned, the final image analysis was done in a batch-processing action across all images. The islet area was also analyzed using basic image analysis software (Image-Pro Plus; Media Cybernetics, Bethesda, MD, USA) by manually drawing the islet perimeter. Glucagon- and somatostatin-positive areas were determined using Image-Pro Plus.

\section{Statistical analysis}

Results are expressed as means \pm s.E.M. Differences between the groups were calculated by Student's $t$-test
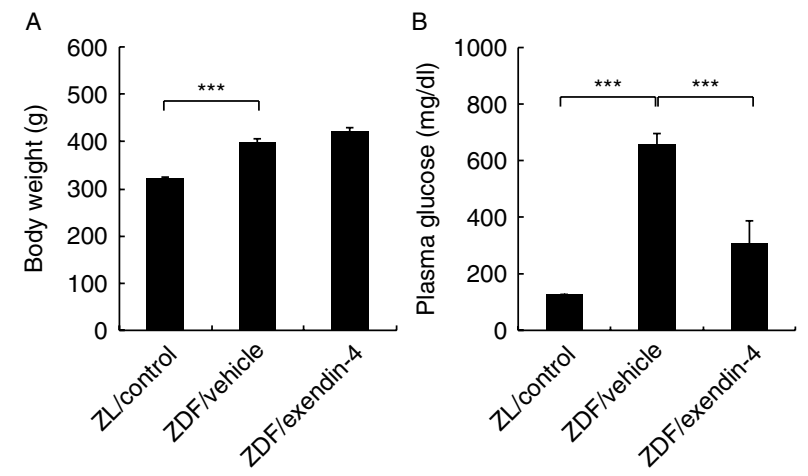

C

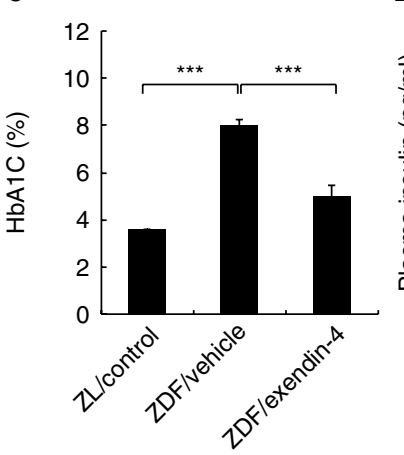

D

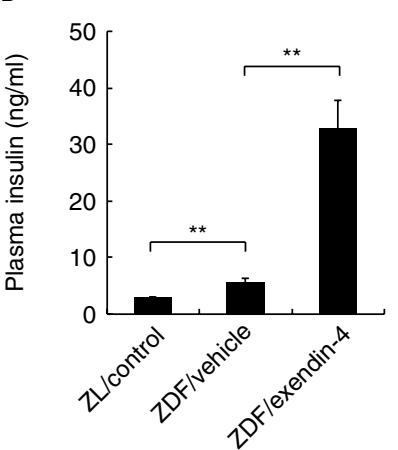

Figure 1

Body weight and blood biochemistry. Body weight (A), plasma glucose concentrations (B), HbA1C (C), and plasma insulin concentrations (D) of non-diabetic control ZL rats and vehicle- or exendin-4-treated ZDF rats at 13 weeks of age. Statistically significant differences are indicated by $* * P<0.01$ and $* * * P<0.001$. Student's $t$-test.

and were considered to be statistically significant when $P<0.05$.

\section{Results}

\section{Body weight}

Body weight data (Fig. 1A) showed that ZDF rats at 13 weeks of age exhibited significant obesity compared with non-diabetic control ZL rats. Exendin-4 treatment yielded no significant difference in the body weight of ZDF rats.

\section{Plasma glucose, HbA1c, and insulin}

ZL rats maintained normoglycemia and normoinsulinemia at 13 weeks of age (Fig. 1B, C and D).

By contrast, the ZDF control group (vehicle treatment) developed overt hyperglycemia and doubled HbA1c levels. Exendin-4 treatment significantly lowered plasma glucose and $\mathrm{HbA1c}$, ameliorating diabetes.

Published by Bioscientifica Ltd 
Vehicle-treated ZDF rats had a significant adaptive hyperinsulinemia, although the approximately twofold increase in insulin secretion seemed insufficient to overcome insulin resistance. Exendin-4-treated ZDF rats displayed dramatic hyperinsulinemia, indicating that exendin- 4 treatment enabled $\beta$-cells to secrete compensatory amounts of insulin to ameliorate diabetes.

\section{Histopathology}

Islets of control ZL rats were round or normal, and displayed no pathology at 13 weeks of age (Fig. 2). Insulin immunostaining in ZL islets was consistently intense and homogeneous. The mantle of glucagon-positive $\alpha$-cells surrounded insulin-positive $\beta$-cells at the islet core. Several somatostatinpositive $\delta$-cells were also observed in the mantle.
Vehicle-treated ZDF islets showed irregular outlines with $\beta$-cell vacuolation, indicative of degenerative change. ZDF islets also showed slight hypertrophy. Insulin immunohistochemistry revealed that ZDF islets had a marked reduction in insulin content, suggesting evidence for insulin degranulation. Glucagon-positive $\alpha$-cells were also decreased. In accordance with disorganized islet structure, the mantle of $\alpha$-cells was disrupted. Glucagonpositive $\alpha$-cells and somatostatin-positive $\delta$-cells were scattered in ZDF islets.

Islets of exendin-4-treated ZDF rats displayed marked hypertrophy. Treatment of ZDF rats with exendin-4 decreased $\beta$-cell vacuolation. However, exendin- 4 treatment had little effect on the irregular shape of the islets. Exendin-4 treatment significantly ameliorated the reduction in insulin immunostaining in ZDF islets,
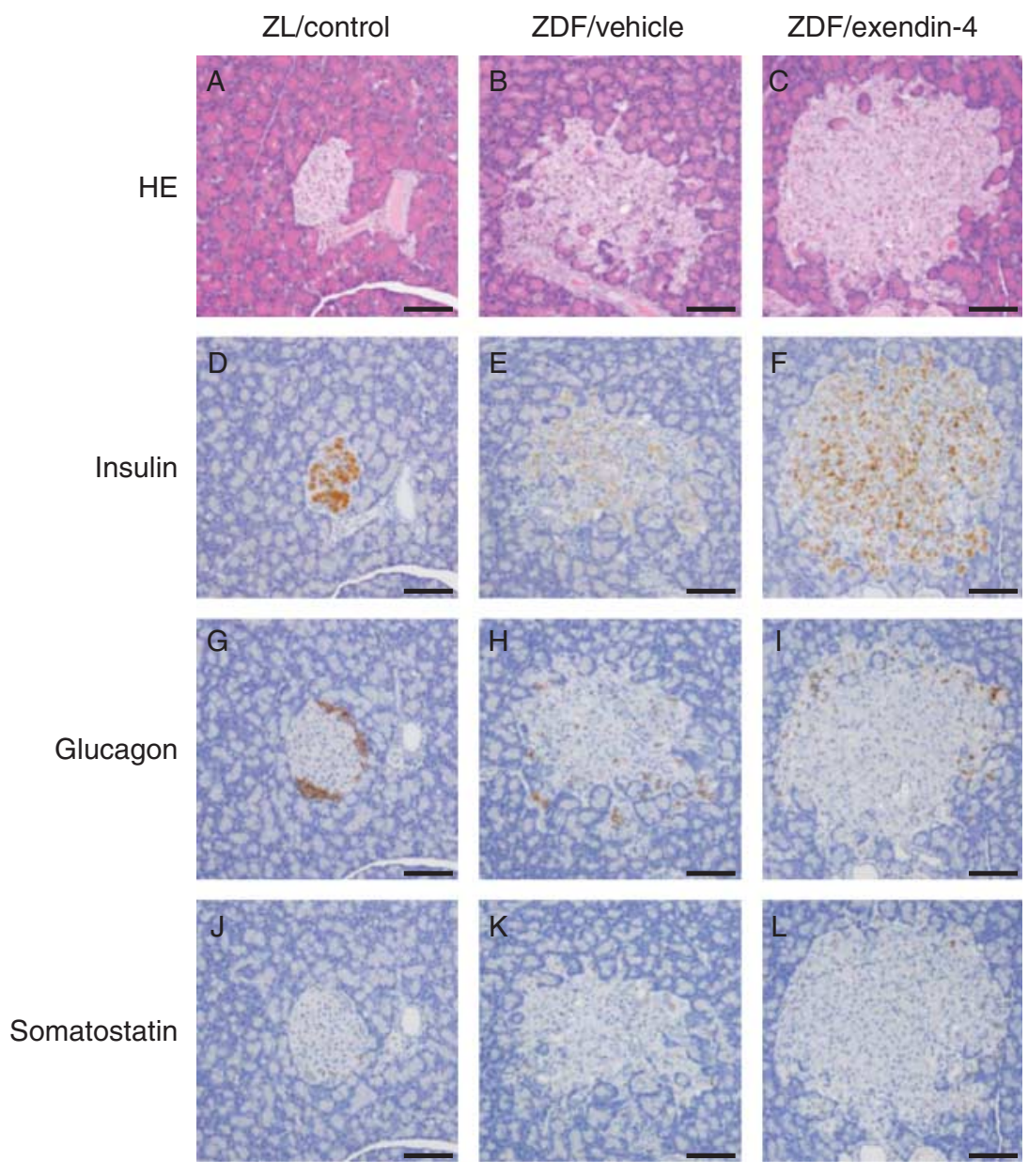

Figure 2

Islets of Langerhans from non-diabetic control ZL (A, D, G and J) and vehicle- $(B, E, H$ and $K)$ or exendin-4-treated $Z D F$ rats $(C, F, I$ and $L)$ at 13 weeks of age. Sections were stained with hematoxylin and eosin

(A, B and C), or immunostained for insulin (D, E and F), glucagon ( $G, H$ and $\mathrm{I}$ ), or somatostatin $(\mathrm{J}, \mathrm{K}$ and $\mathrm{L}$ ) using the diaminobenzidine-based colorization method and hematoxylin counterstaining. Scale bars: $100 \mu \mathrm{m}$.

Published by Bioscientifica Ltd 
consistent with hyperinsulinemia. Exendin-4 treatment did not have a significant effect on either glucagon-positive $\alpha$-cells or somatostatin-positive $\delta$-cells.

\section{Comparison of automated with manual image analysis methods}

We developed automated procedures for quantifying islet area and insulin-positive area in insulin-immunostained pancreas specimens with trainable software, Tissue Studio, to recognize and quantify tissue patterns (Fig. 3). We trained the algorithm to optimally recognize islet area distinct from other pancreatic regions (acinar cells, etc.). This software could also quantify the insulin-positive area.

We compared islet area values determined by automated quantification with those determined by manual quantification (Fig. 4). We traced each islet perimeter by hand to define the islet area in basic image analysis software Image-Pro Plus (Fig. 4A). The method was quite laborious and it took plenty of time to analyze all islets in each pancreas section, which have about 100 islets. An extremely strong positive correlation was observed between the automatic and manual evaluation of islet area (Fig. 4B). These data supported the validity of the automatic quantification method.

\section{Automated image analysis of exendin-4-treated ZDF islets}

We performed automated analysis of the insulin-positive area and islet area of all rats (Fig. 5). The islet area of control ZDF rats was slightly increased compared with that of non-diabetic control ZL rats, although the difference was not statistically significant $(P=0.0505)$. However, the pancreas of ZDF vehicle rats showed a strong decline in insulin-positive area.

Treatment of ZDF rats with exendin-4 markedly increased islet area reflecting strong islet hypertrophy. Exendin-4-treated ZDF islets had strongly increased insulin-positive area as well. Thus, exendin- 4 treatment markedly improved pancreatic insulin store, consistent with overt hyperinsulinemia.

\section{Image analysis of glucagon-positive $\alpha$-cell area and somatostatin-positive $\delta$-cell area}

We also investigated glucagon-positive area and somatostatin-positive area of all rats with Tissue Studio (Fig. 6). Control ZDF rats had significantly less glucagon-positive $\alpha$-cells. Exendin-4 treatment had no significant effect on glucagon-positive $\alpha$-cell area. Somatostatin-positive



Figure 3

Automated image analysis of pancreatic islets. Whole slide images of insulin-immunostained pancreas sections were analyzed by Tissue Studio (A). The software automatically segmented a whole slide image into small image objects (B). We trained the algorithm to optimally distinguish islets (coded orange) from other pancreatic regions (coded blue) by selecting representative small objects and classifying them as islets or other pancreatic regions (C). As a result of training, the algorithm automatically recognized pancreatic islets (coded orange) and quantified their area in batch processing (D). The insulin-positive area (coded yellow) in islets was also automatically quantified (E). Representative images of pancreas specimens from non-diabetic control ZL and vehicle-treated diabetic ZDF rats are shown. Scale bars: $100 \mu \mathrm{m}$.

Published by Bioscientifica Ltd. 
A
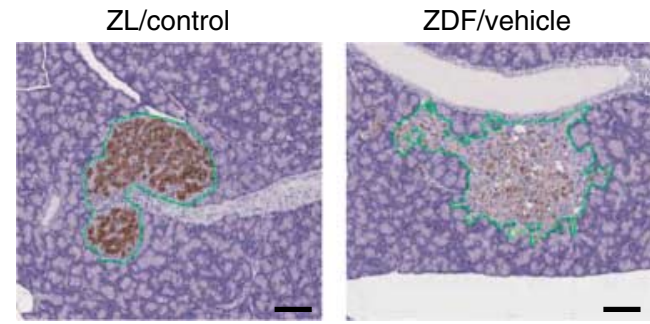

B

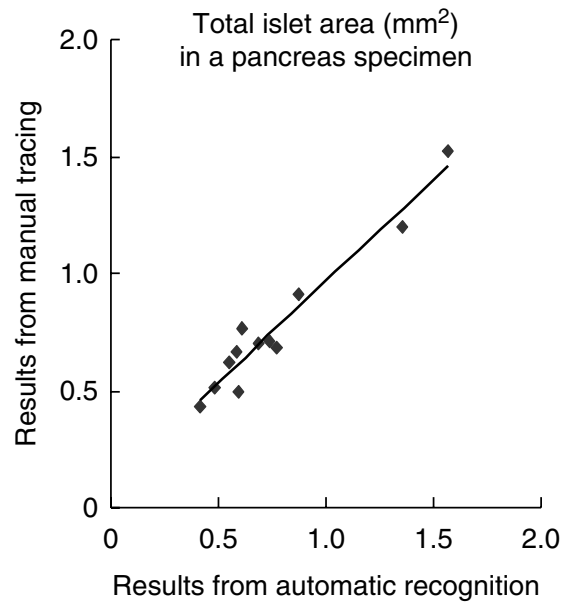

Figure 4

Comparison of automated image analysis with manual image analysis. (A) Islet area of 12 insulin-immunostained pancreas specimens (ZL, ZDF/vehicle, ZDF/exendin-4, four samples from each group) was determined manually by tracing the islet perimeters (green lines). Manual tracing of islet perimeters was quite laborious and time consuming, especially for regions as irregularly shaped as ZDF islets. Representative images of pancreas specimens from non-diabetic control ZL and vehicletreated diabetic ZDF rats are shown. Scale bars: $100 \mu \mathrm{m}$. (B) Total islet area values $\left(\mathrm{mm}^{2}\right)$ in pancreas specimens determined by automatic recognition (Fig. 3) were compared with those determined by manual tracing (A). Data were fitted using linear least-squares fitting analysis. The correlation coefficient between the automatic ( $x$-axis) and manual analysis ( $y$-axis) of islet area was $r=0.971(P<0.001 ; y=0.8624 x+0.1043)$.

$\delta$-cell area was comparable with each other among ZL, vehicle-treated ZDF, and exendin-4-treated ZDF pancreases.

\section{Discussion}

In this study, we have successfully demonstrated the ameliorative effect of long-term exendin- 4 treatment on glucose control and pancreatic histopathology in the diabetic ZDF rat, an animal model with pathophysiological similarities to human type 2 diabetes (Clark et al. 1983, Tokuyama et al. 1995, Pick et al. 1998, Jones et al. 2010). ZDF rats exhibited an impaired adaptation of $\beta$-cell function and suffered severe depletion of stored insulin. ZDF islets also had irregularly shaped structure with a
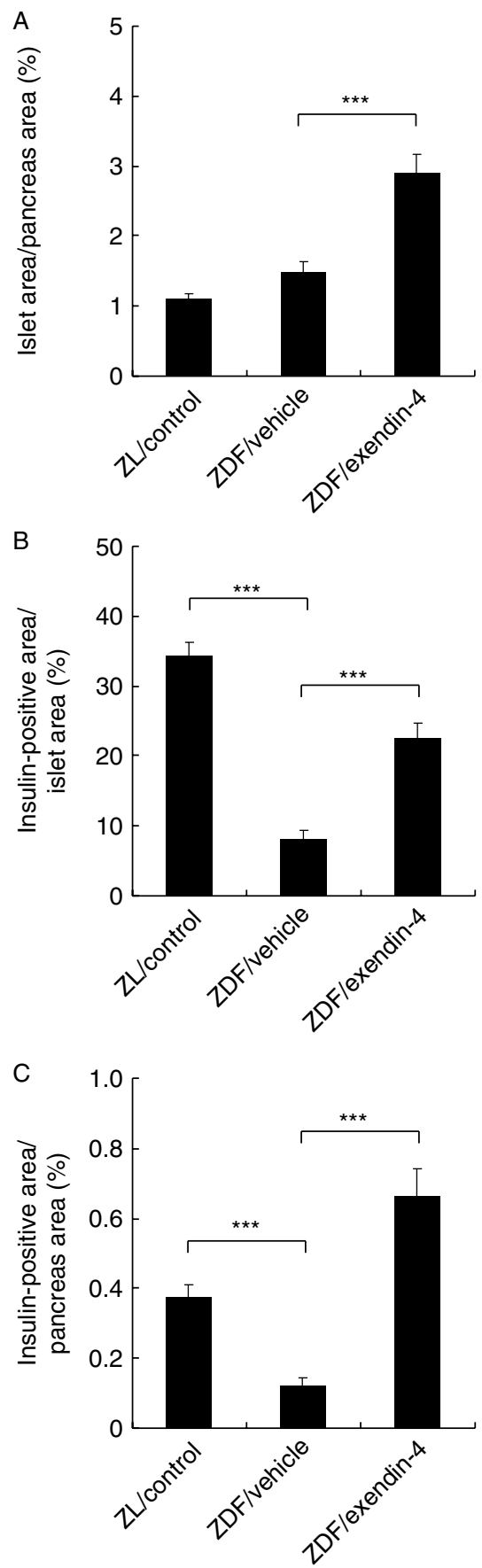

Figure 5

Automated quantitative image analysis of insulin-immunostained pancreases. Pancreatic islet area (A), insulin-positive area in islets (B), and pancreatic insulin-positive area (C) from non-diabetic control ZL rats, and vehicle- or exendin-4-treated ZDF rats at 13 weeks of age are shown. Statistically significant differences are indicated by $* * * P<0.001$. Student's $t$-test. 

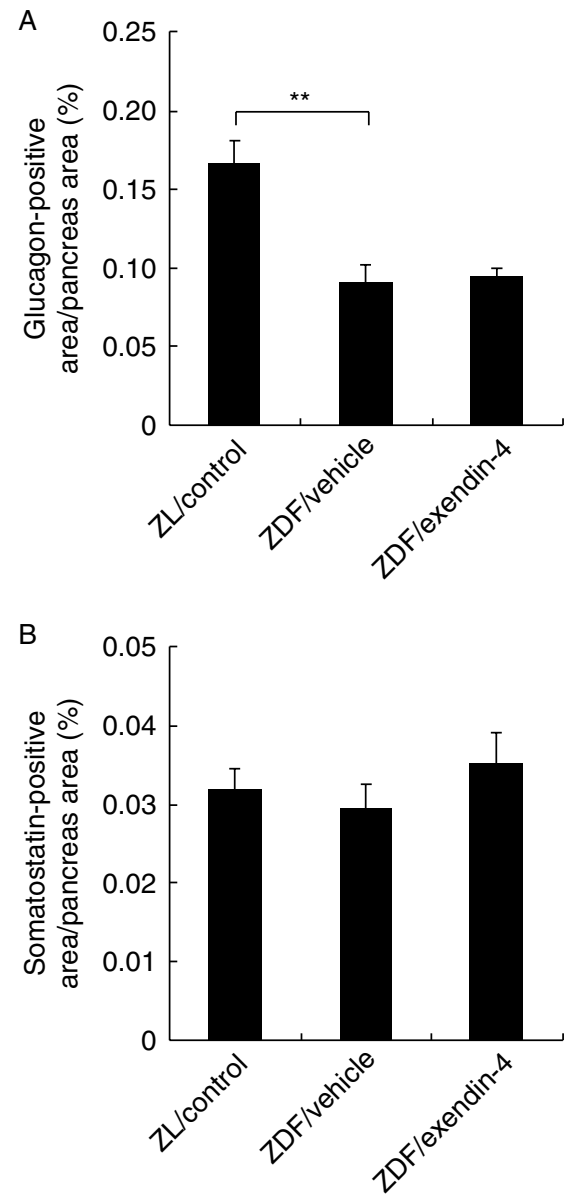

Figure 6

Automated quantitative image analysis of glucagon- and somatostatinimmunostained pancreases. Pancreatic glucagon-positive $\alpha$-cell area (A) and somatostatin-positive $\delta$-cell area from non-diabetic control $Z L$ rats, and vehicle- or exendin-4-treated ZDF rats at 13 weeks of age are shown. Statistically significant differences are indicated by $* * P<0.01$. Student's t-test.

disrupted mantle of $\alpha$-cells. Exendin- 4 treatment markedly increased islet area and insulin-positive area, ameliorating diabetes significantly.

Vehicle-treated ZDF islets showed slight hypertrophy compared with non-diabetic control ZL rats. However, a marked decrease in insulin store caused a substantial reduction in pancreatic stored insulin in ZDF rats. On the other hand, in exendin-4-treated ZDF rat pancreas, the increase in islet area as well as that in insulin content contributed to the restoration of total pancreatic insulin store. These results emphasized the need for quantification of islet area in addition to insulin-positive area for detailed understanding of pancreatic islet histopathology.
Image analysis plays an important role in transforming qualitative histological observations to quantitative characteristics. Development of a digital scanner has enabled whole slide access with high resolution and provided more tissues to be analyzed. Most of the analytic software is pixel-based, one that isolates and quantifies colored pixels such as immunopositive areas (Rojo et al. 2009, Prasad \& Prabhu 2011, Isse et al. 2012). Segregation of specific target tissues is still particularly challenging, such as in the case of ZDF islets (Figs 2, 3 and 4) with a morphologically intricate structure. Manual tracing of target regions is possible, but laborious and time consuming. To overcome limitations of laborious manual quantification of immunostained tissue sections, some automatic tissue recognition software has been developed. In most cases, however, the software can be complex and quite difficult for non-experts. Tissue Studio can recognize microscopic tissue patterns after training by a histologist with no need of good programming knowledge and no need for a custom-made program.

In the present study, we have illustrated a specific application of an automatic trainable software, Tissue Studio, to quantify islet area and insulin-positive area of the pancreas objectively and rapidly. Using Tissue Studio, we successfully recognized irregularly shaped ZDF islets with depleted insulin immunostaining, which are quite difficult to automatically recognize. The correlation between automatic and manual image analysis was extremely good (Fig. 4), corroborating the validity of automatic morphometry analysis. Once the software system was trained, the final image analysis could be performed in a batch-processing action across all images to collect morphometry data without histologists laboriously drawing all islet perimeters in all pancreas sections.

We also studied glucagon-positive $\alpha$-cells. The mantle of $\alpha$-cells was disrupted with scattering $\alpha$-cells as reported previously (Pick et al. 1998). We quantitatively showed that glucagon-positive $\alpha$-cell area was decreased in ZDF islets. We also showed that 8-week exendin-4 treatment had no significant effect on glucagon-positive $\alpha$-cell area in ZDF rats. By contrast, 4-week exendin-4 treatment on SD rats reportedly increased non- $\beta$-cells, most of which were glucagon-positive $\alpha$-cells (Xu et al. 1999). The difference may be due to the diabetic state of exendin4-treated rats. Indeed, 4-week exendin-4 treatment on partially pancreatomized diabetic rats did not significantly increase non- $\beta$-cell mass (Xu et al. 1999).

In conclusion, vehicle-treated ZDF rats developed hyperglycemia by 13 weeks of age. Histological examination revealed that islets of these control

Published by Bioscientifica Ltd. 
ZDF rats were depleted of insulin and exhibited a disorganized architecture. Automated image analysis provided qualitative and quantitative evidence that exendin-4 treatment ameliorated the loss of pancreatic insulin content and gave rise to islet hypertrophy. Simultaneously, extendin-4 restored plasma insulin levels and the control of glucose in ZDF rats. Thus, the application of automated image analysis to pancreatic tissue is feasible and will make a detailed evaluation of pancreatic islet histopathology more rapid and accurate in preclinical drug efficacy studies.

\section{Declaration of interest}

The authors work for Mitsubishi Tanabe Pharma Corporation.

\section{Funding}

This research did not receive any specific grant from any funding agency in the public, commercial or not-for-profit sector.

\section{Acknowledgements}

We thank Ms Yasuko Ogawa for her excellent technical assistance. We also thank Prof. Dr Bernhard F Becker (Ludwig-Maximilians-University, Munich) for restyling our English.

\section{References}

Butler AE, Janson J, Bonner-Weir S, Ritzel R, Rizza RA \& Butler PC 2003 $\beta$-Cell deficit and increased $\beta$-cell apoptosis in humans with type 2 diabetes. Diabetes 52 102-110. (doi:10.2337/diabetes.52.1.102)

Clark JB, Palmer CJ \& Shaw WN 1983 The diabetic Zucker fatty rat. Proceedings of the Society for Experimental Biology and Medicine 173 68-75.

Drucker DJ 2006 The biology of incretin hormones. Cell Metabolism 3 153-165. (doi:10.1016/j.cmet.2006.01.004)

Drucker DJ \& Nauck MA 2006 The incretin system: glucagon-like peptide-1 receptor agonists and dipeptidyl peptidase- 4 inhibitors in type 2 diabetes. Lancet 368 1696-1705. (doi:10.1016/S0140-6736(06)69705-5)

Finegood DT, McArthur MD, Kojwang D, Thomas MJ, Topp BG, Leonard T \& Buckingham RE $2001 \beta$-Cell mass dynamics in Zucker diabetic fatty rats. Rosiglitazone prevents the rise in net cell death. Diabetes $\mathbf{5 0}$ 1021-1029. (doi:10.2337/diabetes.50.5.1021)

Isse K, Lesniak A, Grama K, Roysam B, Minervini MI \& Demetris AJ 2012 Digital transplantation pathology: combining whole slide imaging, multiplex staining and automated image analysis. American Journal of Transplantation 12 27-37. (doi:10.1111/j.1600-6143.2011.03797.x)

Jones HB, Nugent D \& Jenkins R 2010 Variation in characteristics of islets of Langerhans in insulin-resistant, diabetic and non-diabetic-rat strains. International Journal of Experimental Pathology 91 288-301. (doi:10.1111/ j.1365-2613.2010.00713.x)
Kolterman OG, Buse JB, Fineman MS, Gaines E, Heintz S, Bicsak TA, Taylor K, Kim D, Aisporna M, Wang Y et al. 2003 Synthetic exendin-4 (exenatide) significantly reduces postprandial and fasting plasma glucose in subjects with type 2 diabetes. Journal of Clinical Endocrinology and Metabolism 88 3082-3089. (doi:10.1210/jc.2002-021545)

Li Y, Hansotia T, Yusta B, Ris F, Halban PA \& Drucker DJ 2003 Glucagon-like peptide-1 receptor signaling modulates $\beta$ cell apoptosis. Journal of Biological Chemistry 278 471-478. (doi:10.1074/jbc.M209423200)

Pick A, Clark J, Kubstrup C, Levisetti M, Pugh W, Bonner-Weir S \& Polonsky KS 1998 Role of apoptosis in failure of $\beta$-cell mass compensation for insulin resistance and $\beta$-cell defects in the male Zucker diabetic fatty rat. Diabetes 47 358-364. (doi:10.2337/diabetes. 47.3.358)

Prasad K \& Prabhu GK 2011 Image analysis tools for evaluation of microscopic views of immunohistochemically stained specimen in medical research - a review. Journal of Medical Systems 36 2621-2631. (doi:10.1007/s10916-011-9737-7)

Prentki M \& Nolan CJ 2006 Islet $\beta$ cell failure in type 2 diabetes. Journal of Clinical Investigation 116 1802-1812. (doi:10.1172/JCI29103)

Rojo MG, Bueno G \& Slodkowska J 2009 Review of imaging solutions for integrated quantitative immunohistochemistry in the Pathology daily practice. Folia Histochemica et Cytobiologica 47 349-354. (doi:10.2478/ v10042-008-0114-4)

Sakuraba H, Mizukami H, Yagihashi N, Wada R, Hanyu C \& Yagihashi S 2002 Reduced $\beta$-cell mass and expression of oxidative stress-related DNA damage in the islet of Japanese type II diabetic patients. Diabetologia 45 85-96. (doi:10.1007/s125-002-8248-z)

Stoffers DA, Kieffer TJ, Hussain MA, Drucker DJ, Bonner-Weir S, Habener JF \& Egan JM 2000 Insulinotropic glucagon-like peptide 1 agonists stimulate expression of homeodomain protein IDX-1 and increase islet size in mouse pancreas. Diabetes 49 741-748. (doi:10.2337/diabetes.49. 5.741)

Tokuyama Y, Sturis J, DePaoli AM, Takeda J, Stoffel M, Tang J, Sun X, Polonsky KS \& Bell GI 1995 Evolution of $\beta$-cell dysfunction in the male Zucker diabetic fatty rat. Diabetes 44 1447-1457. (doi:10.2337/diabetes. 44.12.1447)

Tourrel C, Bailbé D, Meile MJ, Kergoat M \& Portha B 2001 Glucagon-like peptide- 1 and exendin- 4 stimulate $\beta$-cell neogenesis in streptozotocintreated newborn rats resulting in persistently improved glucose homeostasis at adult age. Diabetes 50 1562-1570. (doi:10.2337/ diabetes.50.7.1562)

Wang Q \& Brubaker PL 2002 Glucagon-like peptide-1 treatment delays the onset of diabetes in 8 week-old db/db mice. Diabetologia 45 1263-1273. (doi:10.1007/s00125-002-0828-3)

Xu G, Stoffers DA, Habener JF \& Bonner-Weir S 1999 Exendin-4 stimulates both $\beta$-cell replication and neogenesis, resulting in increased $\beta$-cell mass and improved glucose tolerance in diabetic rats. Diabetes $\mathbf{4 8}$ 2270-2276. (doi:10.2337/diabetes.48.12.2270)

Yamairi F, Utsumi H, Ono Y, Komorita N, Tanaka M \& Fukunari A 2011 Expression of vascular endothelial growth factor (VEGF) associated with histopathological changes in rodent models of osteoarthritis. Journal of Toxicologic Pathology 24 137-142. (doi:10.1293/tox.24.137)

Yoon KH, Ko SH, Cho JH, Lee JM, Ahn YB, Song KH, Yoo SJ, Kang MI, Cha BY, Lee KW et al. 2003 Selective $\beta$-cell loss and $\alpha$-cell expansion in patients with type 2 diabetes mellitus in Korea. Journal of Clinical Endocrinology and Metabolism 88 2300-2308. (doi:10.1210/jc.2002020735)

Received in final form 4 October 2012

Accepted 22 October 2012

Accepted Preprint published online 22 October 2012 http://www.joe.endocrinology-journals.org DOI: $10.1530 / J O E-12-0456$
C 2013 Society for Endocrinology Printed in Great Britain
Published by Bioscientifica Ltd 